\section{Sur l'existence de solutions de certains nouveaux problèmes pour un système d'équations différentielles hyperboliques du second ordre à deux variables indépendantes}

par Z. SzMYDT (Kraków)

Introduction. Considérons le système de $n$ équations différentielles $u_{x y}^{(i)}(x, y)=f^{(i)}\left(x, y, u^{(1)}, \ldots, u^{(n)}, u_{x}^{(1)}, \ldots, u_{x}^{(n)}, u_{y}^{(1)}, \ldots, u_{y}^{(n)}\right) \quad(i=1,2, \ldots, n)$, aux seconds membres continus, que nous écrirons dans la suite sous la forme vectorielle

$$
\text { (I) } \quad U_{x y}(x, y)=F^{\prime}\left(x, y, U, \delta_{x}, U_{y}\right) \text {. }
$$

Dans la note [4] je me suis occupée d'une généralisation de problèmes classiques relatifs an système (I) et j'y ai introduit un type de problèmes - problèmes du type $T$ - (cf. [4], p. 68, ainsi que le $\$ 1$ de cet article) qui comprenait comme cas particuliers les problèmes de Darboux, de Cauchy, de Picard, de Goursat et les problèmes mixtes. J'ai démontré ([4], Théorème 1, p. 69) l'existence d'une solution d'un problème du type $T$ englobant les problèmes de Darboux et de Cauchy et j'ai esquissé les démonstrations de l'existence d'une solution d'un problème du type $T$ plus général ([4], Problème I, p. 68) à l'étude duquel est consacré cet article.

Du Théorème 1, que nous allons démontrer dans le $\S 4$, résultent immédiatement les Corollaires 1 et 2 (énoncés dans le $\$ 8$ ) assurant l'existence d'une solution des problèmes de Darboux et de Cauchy relatifs au système $(\mathrm{I}){ }^{\left({ }^{1}\right)}$. Du Théorème 3 , démontré dans le $\$ 6$ on déduit le $\mathrm{Co}$ rollaire 3 (cf. §8) assurant l'existence d'une solution du problème de Picard dans des hypothèses assez faibles. Les théorèmes en question donnent aussi une évaluation de la solution $U(x, y)$ et de ses dérivées partielles.

Le Corollaire 1 généralise le théorème de $\mathrm{P}$. Frartman et $\mathrm{A}$. Wintner concernant l'existence d'une solution du problème de Darboux ([1], (I), p. 836), l'hypothèse que la fonction $F(x, y, U, P, Q)$ satisfait à la condition de Lipschitz par rapport aux variables $P$ et $Q$ y étant remplacée

(1) Les Corollaires 1 et 2 résultent aussi du théorème 1 de la note [4] qui est lui-même une conséquence du Théorème 1 de cet article. par l'Hypothèse $K$, moins restrictive (cf. § 3). Je dois à A. Pliś l'idée d'introduire cette hypothèse de régularité.

La méthode qui sert à démontrer l'existence d'une solution des problèmes du type $T$ considérés dans cet article est basée sur le théorème de Schauder ([3], Satz 1, p. 173), appliqué par S. Mazur (cf. § 9, Remarque 6) à la démonstration du résultat de P. Hartman et $\mathrm{A}$. Wintner cité ci-dessus (Séance du Groupe d'Analyse de l'Institut Mathématique de l'Académie Polonaise des Sciences, Varsovie 1954).

\$1. Désignons par $D$ le rectangle défini par les inégalités (1.1) $-\alpha \leqslant x \leqslant \alpha, \quad-\beta \leqslant y \leqslant \beta, \quad$ où $\quad 0<\alpha<\infty, \quad 0<\beta<\infty$. Considérons deux courbes continues $\Gamma$ et $\Lambda$ définies respectivement par les équations

$$
\begin{array}{lll}
y=\gamma(x) & \text { lorsque } & -\alpha \leqslant x \leqslant \alpha \\
x=\lambda(y) & \text { lorsque } & -\beta \leqslant y \leqslant \beta
\end{array}
$$

et situées dans le rectangle $D$.

Soit

$$
\begin{array}{ll}
U=\left(u^{(1)}, \ldots, u^{(n)}\right), & P=\left(p^{(1)}, \ldots, p^{(n)}\right), \\
Q=\left(q^{(1)}, \ldots, q^{(n)}\right), & F=\left(f^{(1)}, \ldots, f^{(n)}\right), \\
U_{x}=P, & U_{y}=Q .
\end{array}
$$

Considérons le système de $n$ équations différentielles écrit sous la forme vectorielle

$$
U_{x y}(x, y)=F(x, y, \delta, P, Q),
$$

où $F(x, y, U, P, Q)$ est une fonction continue dans un ensemble de l'espace $x, y, U, P, Q$. Appelons problème du type $T$ (cf. [4], p. 68) tout problème concernant l'existence d'une solution $U(x, y)$ du système (1.3) ayant des dérivées $U_{x}, U_{y}, U_{x y}$ continues dans le rectangle $D$ et satisfaisant à certaines relations données le long des deux courbes $\Gamma$ et $\Lambda$.

On vérifie facilement que tous les problèmes classiques (problèmes de Darboux, de Cauchy, de Goursat, de Picard et problèmes mixtes) sont des problèmes du type $T$. Voici maintenant deux problèmes du type $T$ plus généraux $\left(^{2}\right)$ :

ProBlÈme I. Existe-t-il une solution $U(x, y)$ du systèrre (1.3) ayant des dérivées $U_{x}, U_{y}, J_{x y}$ continues dans le rectangle $D$ et satisfaisant aux conditions: (1.4) $\quad U\left(x_{0}, y_{0}\right)=\stackrel{U}{U}$,

(1.5) $\quad U_{x}(x, y)=G\left[x, U(x, y), U_{y}(x, y)\right] \quad$ lorsque $y=\gamma(x)$

$$
\text { et }-\alpha \leqslant x \leqslant a \text {, }
$$

(2) Cf. [4], p. 68. Le Problème II a été énoncé dans la note [4] sous une forme un peu moins générale, la fonction $B$ ne dépendant que de $y$. 


$$
\begin{aligned}
& U_{y}(x, y)=H\left[y, \dot{U}(x, y), U_{x}(x, y)\right] \quad \text { lorsque } \quad x=\lambda(y) \\
& \text { et }-\beta \leqslant y \leqslant \beta \text {, }
\end{aligned}
$$

où $\left(x_{0}, y_{0}\right)$ est un point arbitraire de $D$, Uீ un vecteur constant et $G(x, U, Q)$, $H(y, U, P)$ sont des fonctions continues données d'avance?

PROBLÈme II. Existe-t-il une solution $U(x, y)$ du système (1.3) ayant des dérivées $U_{x}, U_{y}, D_{x y}$ continues dans le rectangle $D$ et satisfaisant aux conditions:

$U_{x}(x, y)=G\left[x, U(x, y), U_{y}(x, y)\right]$ lorsque $y=\gamma(x) \quad$ et $\quad-\alpha \leqslant x \leqslant \alpha$, $U(\lambda(y), y)=U^{r}+\int_{y_{0}}^{y} B\left[t, U(\lambda(t), t), U_{x}(\lambda(t), t)\right] d t \quad$ lorsque $\quad-\beta \leqslant y \leqslant \beta$,

oì $G(x, U, Q)$ et $B(y, U, P)$ sont des fonctions continues données d'avance, Uீ est un vecteur constant et $y_{0}$ un point arbitraire de l'intervalle $\langle-\beta, \beta\rangle$ ?

Remarque 1 . Si l'on admet que $\lambda(y)$ est une fonction de classe $C^{1}$, le Problème II est équivalent au Problème $I$ dans lequel

$$
x_{0}=\lambda\left(y_{0}\right), \quad H(y, U, P)=B(y, U, P)-\lambda^{\prime}(y) P .
$$

Notre remarque résulte immédiatement de l'identité

$$
\frac{d}{d y} U(\lambda(y), y)=\lambda^{\prime}(y) U_{x}(\lambda(y), y)+U_{y}(\lambda(y), y)
$$

satisfaite par chaque fonction $U(x, y)$ de classe $C^{\mathrm{x}}$.

DÉFINITION 1. Soit $V=\left(v^{(1)}, \ldots, v^{(n)}\right)$ un vecteur arbitraire. Le symbole $|\nabla|$ désignera la norme du vecteur $V=\left(v^{1)}, \ldots, v^{(n)}\right)$ donnée par la formule

$$
|V|=\max _{1 \leqslant i \leqslant n}\left|v^{(i)}\right| .
$$

Dans cet article nous démontrerons les Théorèmes 1 et 2 sur l'existence d'une solution du Problème I. L'une des hypothèses intervenant dans ces théorèmes suppose que (cf. $\S 3$, Hypothèse $A_{\mathfrak{I}}, 4^{0}$ )

(1.8) $|H(y, U, P)| \leqslant c<r \quad$ lorsque $-\beta \leqslant y \leqslant \beta, \quad|U| \leqslant r, \quad|P| \leqslant r$, où $0<\beta<\infty, 0<r<\infty$. Par conséquent, bien que le Problème II dans lequel $B(y, U, P) \equiv 0$ puisse être considéré comme un cas particulier du Problème I (cf. Remarque 1), l'existence de sa solution ne résulte pas des Théorèmes 1 ou 2 dans l'hypothèse que la fonction $\lambda(y)$ est une fonction arbitraire de classe $C^{\mathbf{1}}$. En effet, il faudrait alors admettre (cf. (1.7), (1.8))

$$
\text { (1.9) } \quad\left|\lambda^{\prime}(y)\right|<1 \text { lorsque }-\beta \leqslant y \leqslant \beta \text {. }
$$

Cependant l'hypothèse (1.9) n'est pas essentielle pour l'existence d'une so- lution du Problème II. O'est pourquoi le Problème II sera aussi considéré indépendamment du Problème I. Les démonstrations des théorèmes 3 et 4 concernant l'existence d'une solution du Problème II, au fond analogues à celles des Théorèmes 1 et 2 , seront esquissées dans le $\S 6$.

§ 2. La démonstration de l'existence d'une solution du Problème I sera basée sur le Lemme 1 et sur la Remarque 2 que voici:

LEMME 1. L'existence d'une solution du Problème I résulte de celle d'une solution de classe $C^{1} d u$ système

$$
U=L[U],
$$

où l'opérateur $L[U]$ est défini par la formule

$$
\begin{aligned}
L[U]=U^{2} & +\int_{x_{0}}^{x} G\left[s, U(s, \gamma(s)), U_{y}(s, \gamma(s))\right] d s+ \\
& +\int_{y_{0}}^{y} H\left[t, U(\lambda(t), t), U_{x}(\lambda(t), t)\right] d t+ \\
& +\int_{x_{0}}^{x}\left\{\int_{\gamma(s)}^{y_{0}} F\left[s, t, U(s, t), U_{x}(s, t), U_{y}(s, t)\right] d t\right\} d s+ \\
& +\int_{y_{0}}^{y}\left\{\int_{\lambda(t)}^{x} F\left[s, t, U(s, t), U_{x}(s, t), U_{y}(s, t)\right] d s\right\} d t .
\end{aligned}
$$

Démonstration. Chaque solution $U(x, y)$ de classe $C^{1}$ de l'équation (2.1) satisfait aux relations

$$
\begin{aligned}
& U_{x}(x, y)=G\left[x, U(x, \gamma(x)), U_{y}(x, \gamma(x))\right]+ \\
& +\int_{\gamma(x)}^{\nu} F\left[x, t, U(x, t), U_{x}(x, t), U_{y}(x, t)\right] d t, \\
& U_{y}(x, y)=H\left[y, U(\lambda(y), y), U_{x}(\lambda(y), y)\right]+ \\
& +\int_{\lambda(y)}^{x} F\left[s, y, U(s, y), U_{x}(s, y), U_{y}(s, y)\right] d s .
\end{aligned}
$$

On voit immédiatement que les relations (1.5), (1.6) résultent respectivement des relations (2.3) et $(2.4)$ et que (1.4) résulte de (2.1), (2.2). L'équation (1.3) peut être obtenue de chacune des relations $(2.3)$ et $(2.4)$ (cf. (1.2)). Elle met en évidence la continuité de $U_{x y}(x, y)$, car $F(x, y$, $U, P, Q)$ est, par hypothèse, une fonction continue et $U(x, y)$ une fonction de classe $C^{1}$.

Remarque 2. L'existence d'une solution de classe $C^{1}$ du système (2.1) résulte de celle d'un point fixe de la transformation 


$$
U=L[U], \quad \text { où } L[U] \text { est donné par (2.2), }
$$

définie dans un ensemble de l'espace $C^{\mathbf{1}}$. Le théorème de Schauder (cf. [3], Satz 1, p. 173) nous permettra de démontrer, moyennant certaines hypothèses, l'existence du point fixe en question.

§ 3. Notations et hypothèses. Désignons par $\Pi$ l'ensemble défini par les inégalités (cf. $\$ 1$, Définition 1)

$$
\begin{gathered}
-\alpha \leqslant x \leqslant \alpha, \quad-\beta \leqslant y \leqslant \beta, \quad \text { où } \quad \begin{array}{c}
0<\alpha<\infty \\
\quad 0<\beta<\infty,
\end{array} \\
|D| \leqslant r, \quad|P| \leqslant r \quad \text { et }|Q| \leqslant r, \quad \text { où } \quad 0<r<\infty,
\end{gathered}
$$

et considérons en outre les deux ensembles suivants:

$$
\begin{aligned}
& -a \leqslant x \leqslant \alpha, \quad|U| \leqslant r, \quad|Q| \leqslant r, \\
& -\beta \leqslant y \leqslant \beta, \quad|U| \leqslant r, \quad|P| \leqslant r .
\end{aligned}
$$

$$
h=2 \max (\alpha, \beta) .
$$

Tenant compte des notations introduites ci-dessus nous énonçons l'hypothèse suivante:

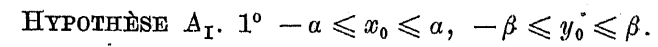

$2^{0} \gamma(x)$ et $\lambda(y)$ sont des fonctions continues telles que

$$
\begin{array}{lll}
-\beta \leqslant \gamma(x) \leqslant \beta & \text { lorsque } & -\alpha \leqslant x \leqslant \alpha, \\
-\alpha \leqslant \lambda(y) \leqslant \alpha & \text { lorsque } & -\beta \leqslant y \leqslant \beta .
\end{array}
$$

$3^{\circ}$ La fonction $F(x, y, U, P, Q)$ continue dans l'ensemble $I I$ défini par (3.1) satisfait à la condition $N \leqslant \min \left\{r / h, r / h^{2}\right\}$, ò̀

$$
N=\max _{\boldsymbol{H} .}|\vec{F}|, \quad \max _{\boldsymbol{I}}=\max _{(x, y, U, P, Q) \in \dot{I}}
$$

$4^{\circ}$ Le vecteur constant $Z^{2}$ et les fonctions vectorielles continues $G, H$ satisfont aux conditions: $\left|U^{\circ}\right| \leqslant c,|G(x, U, Q)| \leqslant c$ dans l'ensemble (3.2), $|H(y, U, P)| \leqslant c$ dans l'ensemble (3.3), où (cf. (3.5)) $c=\min \{r-h N$, $\left.\left(r-N h^{2}\right) /(1+2 h)\right\}$.

HYPOTHÈsE K. La fonction $F(x, y, U, P, Q)$ continue dans l'ensemble II y satisfait d̀ la condition

$|F(x, y, J, P, Q)-F(x, y, U, \tilde{P}, \tilde{Q})| \leqslant \omega_{1}(y,|P-\tilde{P}|, x)+\omega_{2}(x,|Q-\tilde{Q}|, y)$, où $\omega_{1}(y, v, x), \omega_{2}(x, v, y)$ sont des fonctions continues, assujetties dans l'ensemble: $-\alpha \leqslant x \leqslant \alpha,-\beta \leqslant y \leqslant \beta, v \geqslant 0$ aux conditions suivantes:

$1^{0} \omega_{1}(y, v, x), \omega_{2}(x, v, y)$ sont non décroissantes par rapport $\grave{a} v$.

$2^{\circ}$ Pour tout $x$ fixé dans l'intervalle $\langle-\alpha, \alpha\rangle$ et pour tout $\tilde{y},-\beta \leqslant \tilde{y} \leqslant \beta$,

$$
v(y) \equiv 0^{-} \quad \text { lorsque } \quad-\beta \leqslant y \leqslant \beta
$$

est une intégrale unique, passant par le point $(\tilde{y}, 0)$, de chacune des deux équations

$$
\begin{aligned}
& d v / d y=\omega_{1}(y, v, x), \\
& d v / d y=-\omega_{1}(y, v, x) .
\end{aligned}
$$

$3^{\circ}$ Pour tout y fixé dans l'intervalle $\langle-\beta, \beta\rangle$ et pour tout $\tilde{x},-\alpha \leqslant \tilde{x} \leqslant \alpha$

$$
v(x) \equiv 0 \quad \text { lorsque }-\alpha \leqslant x \leqslant a
$$

est une intégrale unique, passant par le point $(\tilde{x}, 0)$, de chacune des deux équations

$$
\begin{aligned}
& d v / d x=\omega_{2}(x, v, y) \\
& d v / d x=-\omega_{2}(x, v, y) .
\end{aligned}
$$

§4. THÉrìme 1. Admettons les hypothèses $\mathrm{A}_{\mathbf{I}}$ et $\mathrm{K}$ et supposons remplie l'une des deux hypothèses suivantes:

a. La fonction $G$ ne dépend pas de la variable $Q$ ou la fonction $\gamma(x)$ est constante.

b. La fonction $H$ ne dépend pas de $P$ ou $\lambda(y)$ est constante.

Dans oes hypothèses il existe une solution du Problème $\mathrm{I}\left({ }^{3}\right)$.

Démonstration. L'hypothèse $b$ jouant relativement au Problème $I$ le même rôle que l'hypothèse a (cf. (1.5) et (1.6)) il est évident qu'il suffit de démontrer le Théorème 1 dans le cas, où l'hypothèse a est satisfaite.

Considérons (cf. Remarque 2) dans l'espace $C^{\mathrm{I}}$. l'ensemble $Z$ des fonctions $U(x, y)$ de classe $O^{1}$ dans le rectangle $D$ (cf. (1.1)) ayant les propriétés suivantes (cf. (3.1) et (3.5))

$$
\begin{aligned}
& |U(x, y)| \leqslant r, \\
& \left.\left|U_{x}(x, y)\right| \leqslant r,\right\} \text { lorsque }(x, y) \in D, \\
& \left|U_{v}(x, y)\right| \leqslant r \\
& U_{x}(x, y+\varepsilon)-U_{x}(x, y) \mid \leqslant N \varepsilon \quad \text { lorsque } \quad(x, y) \epsilon D \\
& (x, y+\varepsilon) \epsilon D, \quad \varepsilon \geqslant 0
\end{aligned}
$$

(3) Cette solution satisfait évidemment aux relations:

$|U(x, y)| \leqslant r, \quad\left|\dot{U}_{x}(x, y)\right| \leqslant r, \quad\left|U_{y}(x, y)\right| \leqslant r, \quad\left|U_{x y}(x, y)\right| \leqslant N$ dans le rectangle $D$ (cf. (1.2) et Hypothèse $A_{I}, 3^{\circ}$ ). 
$(4.5)$

$$
\left|U_{y}(x+\varepsilon, y)-U_{y}(x, y)\right| \leqslant N \varepsilon \quad \text { lorsque } \begin{array}{r}
(x, y) \epsilon D, \\
(x+\varepsilon, y) \epsilon D, \quad \varepsilon \geqslant 0,
\end{array}
$$

$$
\begin{aligned}
\left|U_{x}(x+\varepsilon, y)-U_{x}(x, y)\right| \leqslant \psi(y, x, \varepsilon) \quad \begin{array}{l}
\text { lorsque } \quad(x, y) \in D, \\
(x+\varepsilon, y) \in D, \quad \varepsilon \geqslant 0,
\end{array}
\end{aligned}
$$

$$
\begin{aligned}
\left|U_{y}(x, y+\varepsilon)-U_{y}(x, y)\right| \leqslant \varphi(x, y, \varepsilon) \quad \text { lorsque } \quad(x, y) \epsilon D, & (x, y+\varepsilon) \epsilon D, \quad \varepsilon \geqslant 0,
\end{aligned}
$$

où $\psi(y, x, \varepsilon), \varphi(x, y, \varepsilon)$ sont des fonctions convenablement définies pour $(x, y) \epsilon D$ et $\varepsilon$ positifs suffisamment petits. Passons à leurs définitions. Soit $\delta(\varepsilon)$ le module de continuité $\left.{ }^{4}\right)$ commun aux fonctions $F, G, H$, $\gamma, \lambda$ dans leurs domaines d'existence. Posons

$$
\text { (4.8) } \quad \sigma(\varepsilon)=\max \{\delta(\varepsilon)+\delta(r \varepsilon+r \delta(\varepsilon)), \delta(\varepsilon)+\delta(r \varepsilon)+\delta(N \varepsilon)\}
$$

et soit

$$
\mu(\varepsilon)=\sigma(\varepsilon)+N \delta(\varepsilon)+h\{\delta(\varepsilon)+\delta(r \varepsilon)+\delta(N \varepsilon)\} .
$$

Il résulte des propriétés de la fonction $\delta(\varepsilon)$ (cf. le renvoi $\left({ }^{4}\right)$ ) que

$$
\mu(\varepsilon) \rightarrow 0 \text { pour } \quad \varepsilon \rightarrow 0 .
$$

Désignons par $v=\chi_{1}(y, \xi, \eta ; x)$ une intégrale quelconque de l'équation (3.7) ( $x$ fixé dans l'intervalle $\langle-\alpha, \alpha\rangle$ ) issue du point $(\xi, \eta)$ et par $v=\chi_{2}(y, \xi, \eta ; x)$ celle de l'équation (3.8) passant par le même point. Posons formellement

$$
\text { (4.11) } \quad \psi(y, x, \varepsilon)=\left\{\begin{array}{lll}
\chi_{\mathrm{i}}(y, \gamma(x), \mu(\varepsilon) ; x) & \text { lorsque } & y \geqslant \gamma(x), \\
\chi_{\mathrm{i}}(y, \gamma(x), \mu(\varepsilon) ; x) & \text { lorsque } & y<\gamma(x) .
\end{array}\right.
$$

En tenant compte des propriétés des équations (3.7) et (3.8) et de la relation (4.10), on démontre ([2], Satz 3, p. 149) que $\psi(y, x, \varepsilon)$ est une fonction définie pour $(x, y) \epsilon D$ et $\varepsilon \geqslant 0$ suffisamment petit et que

$$
(4.12) \quad \psi(y, x, \varepsilon) \underset{s \rightarrow 0}{\Rightarrow 0} \text { dans le rectangle } D \text {. }
$$

Posons

(4.13)

$$
\varrho(\varepsilon)=\operatorname{sip}_{-\beta \leqslant y \leqslant \beta} \delta(N \varepsilon+\psi(y, \lambda(y),|\lambda(y+\varepsilon)-\lambda(y)|))
$$

et soit

$$
\nu(\varepsilon)=\mu(\varepsilon)+\varrho(\varepsilon) .
$$

(4) $R(\Xi)$ étant une fonction arbitraire (vectorielle ou non), definie dans un ensemble $\Omega$, une fonction non décroissante $\delta(\varepsilon)$ est dite module de continuité de la fonction $R(\Xi)$ lorsque $\delta(\varepsilon) \rightarrow 0$ pour $\varepsilon \rightarrow 0$ et que $\delta(\varepsilon) \geqslant \sup |R(\Xi)-R(\hat{\Xi})|$ pour tous les $\Xi, \hat{\Xi}_{e} \Omega$ tels que $|\Xi-\hat{\Xi}| \leqslant \varepsilon$
Il résulte des propriétés des fonctions $\mu, \delta$ et $\psi$ énumerées ci-dessus que

$$
\nu(\varepsilon) \rightarrow 0 \quad \text { lorsque } \quad \varepsilon \rightarrow 0 .
$$

Désignons par $\varkappa_{1}(x, \xi, \eta ; y), \varkappa_{2}(x, \xi, \eta ; y)$ les fonctions qui jouent relativement aux équations (3.9) et (3.10) le même rôle que les fonctions $\chi_{1}(y, \xi, \eta ; x)$ et $\chi_{2}(y, \xi, \eta ; x)$ par rapport à $(3.7)$ et $(3.8)$.

Posons formellement

(4.15) $\quad \varphi(x, y, \varepsilon)=\left\{\begin{array}{lll}\varkappa_{1}(x, \lambda(y), \nu(\varepsilon) ; y) & \text { lorsque } & x \geqslant \lambda(y), \\ x_{i}(x, \lambda(y), v(\varepsilon) ; y) & \text { lorsque } & x<\lambda(y) .\end{array}\right.$

On vérifie que la fonction $p(x, y, \varepsilon)$ est définie pour $(x, y) \in D$ et $\varepsilon$ suffisamment petits et que

$$
\varphi(x, y, \varepsilon) \underset{\varepsilon \rightarrow 0}{\Rightarrow} 0 \text { dans le rectangle } D .
$$

Les fonctions $\psi(y, x, \varepsilon)$ et $\varphi(x, y, \varepsilon)$ étant ainsi définies (cf. (4.11) et (4.15)) remarquons qu'elles satisfont aux relations

$$
\begin{aligned}
& \left|\int_{\gamma(x)}^{y} \omega_{1}[t, \psi(t, x, \varepsilon), x] d t\right|=\psi(y, x, \varepsilon)-\mu(\varepsilon), \\
& \left|\int_{\lambda(\nu)}^{x} \omega_{2}[s, \varphi(s, y, \varepsilon), y] d s\right|=\varphi(x, y, \varepsilon)-\nu(\varepsilon)
\end{aligned}
$$

dont nous nous servirons dans la suite.

Nous allons démontrer que (2.5) définit une transformation qui transforme l'ensemble $Z$ (cf. (4.1)-(4.7)) en un sous ensemble de celui-ci.

En effet, d'après les relations (2.5) et $(2.2)$ il est évident que $\tilde{U}(x, y)$ est une fonction de classe $O^{1}$ dans le rectangle $D$ et que $\left({ }^{5}\right)$

$$
\begin{aligned}
& \tilde{U}_{x}(x, y)=G\left[x, U(x, \gamma(x)), U_{y}(x, \gamma(x))\right]+ \\
& +\int_{\gamma(x)}^{y} F\left[x, t, U(x, t), U_{x}(x, t), U_{y^{\prime}}(x, t)\right] d t, \\
& \tilde{U}_{y}(x, y)=H\left[y, U(\lambda(y), y), \sigma_{x}(\lambda(y), y)\right]+ \\
& +\int_{\lambda(y)}^{x} F\left[s, y, U(s, y), U_{x}(s, y), U_{y}(s, y)\right] d s .
\end{aligned}
$$

(5) Dans le cas de l'hypothèse que la fonction $G$ ne dépend pas de la variable $Q$; on doit remplacer partout $G(x, U, Q)$ par $G(x, U)$. 
En tenant compte de (3.4) et de l'Hypothèse $\mathrm{A}_{\mathrm{I}}$ on déduit des relations (2.5), (4.19) et $(4.20)$ les inégalités

$$
\begin{gathered}
|\tilde{U}(x, y)| \leqslant c+2 h c+h^{2} N \leqslant r \\
\left|\tilde{U}_{x}(x, y)\right| \leqslant c+h N \leqslant r, \quad\left|\tilde{U}_{y}(x, y)\right| \leqslant c+h N \leqslant r,
\end{gathered}
$$

ainsi que les inégalités qu'on obtient de (4.4), (4.5) en y remplaçant $U$ par $\tilde{U}$. Pour démontrer que la fonction $\tilde{U}(x, y)$ remplit aussi l'inégalité (4.6) on va profiter des relations

(4.21) $|U(x+\varepsilon, y)-U(x, y)| \leqslant r \varepsilon, \quad\rfloor U(x, y+\varepsilon)-U(x, y) \mid \leqslant r \varepsilon$

qui sont une conséquence de (4.2), (4.3) (cf. la Définition 1 ). $\delta(\varepsilon)$ désignant le module de continuité des fonctions $\gamma, G, F$, on démontre, en s'appuyant sur (4.21), (4.5), (4.8) et sur l'hypothèse a de notre théorème, que

$$
\begin{array}{r}
\text { (4.22) } \quad \mid G\left[x+\varepsilon, U(x+\varepsilon, \gamma(x+\varepsilon)), U_{y}(x+\varepsilon, \gamma(x+\varepsilon))\right]- \\
-G\left[x, U(x, \gamma(x)), U_{y}(x, \gamma(x))\right] \mid \leqslant \sigma(\varepsilon),
\end{array}
$$

et, en tenant compte de (4.21), (4.5), (3.6) et (4.6) on obtient

$$
\begin{aligned}
\mid F[x+\varepsilon, t, U(x+\varepsilon, t), & \left.U_{x}(x+\varepsilon, t), U_{y}(x+\varepsilon, t)\right]- \\
& -F\left[x, t, U(x, t), U_{x}(x, t), U_{y}(x, t)\right] \mid
\end{aligned}
$$$$
\leqslant \delta(\varepsilon)+\delta(r \varepsilon)+\delta(N \varepsilon)+\omega_{1}[t, \psi(t, x, \varepsilon), x] .
$$

Il résulte de (4.19) en vertu de (4.22), (3.5), (4.23), (3.4), (4.9) et (4.17) que

$$
\begin{aligned}
& \left.\mid \tilde{U}_{x}{ }^{\prime} x+\varepsilon, y\right)-\tilde{U}_{x}(x, y) \mid \leqslant \sigma(\varepsilon)+N \delta(\varepsilon)+h\{\delta(\varepsilon)+\delta(r \varepsilon)+\delta(N \varepsilon)\}+ \\
& \quad+\left|\int_{\gamma(x)}^{y} \omega_{1}[t, \psi(t, x, \varepsilon), x] d t\right|=\mu(\varepsilon)+\left|\int_{\gamma(x)}^{y} \omega_{1}[t, \psi(t, x, \varepsilon), x] d t\right|=\psi(y, x, \varepsilon)
\end{aligned}
$$

c'est-à-dire, la fonction $\tilde{U}(x, y)$ satisfait à l'inégalité (4.6). Pơur démontrer qu'elle satisfait aussi à (4.7) nous allons profiter des inégalités:

$$
\text { (4.24) }
$$

$$
\begin{aligned}
& H\left[y+\varepsilon, U(\lambda(y+\varepsilon), y+\varepsilon), U_{x}(\lambda(y+\varepsilon), y+\varepsilon)\right]- \\
& -H\left[y, U(\lambda(y), y), U_{x}(\lambda(y), y)\right] \\
& \leqslant \delta(\varepsilon)+\delta(r \varepsilon+r \delta(\varepsilon))+\delta(N \varepsilon+\psi(y, \lambda(y),|\lambda(y+\varepsilon)-\lambda(y)|)) \\
& \leqslant \delta(\varepsilon)+\delta(r \varepsilon+r \delta(\varepsilon))+\varrho(\varepsilon)
\end{aligned}
$$

(cf. $(4.13))$ et

$$
\begin{gathered}
\text { (4.25) } \quad \mid F\left[s, y+\varepsilon, U(s, y+\varepsilon), U_{x}(s, y+\varepsilon), U_{y}(s, y+\varepsilon)\right]- \\
\quad-F\left[s, y, U(s, y), U_{x}(s, y), U_{\nu}(s, y)\right] \mid \\
\leqslant \delta(\varepsilon)+\delta(r \varepsilon)+\delta(N \varepsilon)+\omega_{2}(s, \varphi(s, y, \varepsilon), y) .
\end{gathered}
$$

Il résulte de (4.20), en vertu de (4.24), (3.4), (3.5), (4.25), (4.8), (4.9), (4.14) et (4.18), que

$$
\begin{aligned}
&\left|\tilde{U}_{y}(x, y+\varepsilon)-\tilde{U}_{y}(x, y)\right| \leqslant \delta(\varepsilon)+\delta(r \varepsilon+r \delta(\varepsilon))+\varrho(\varepsilon)+ \\
&+N \delta(\varepsilon)+h\{\delta(\varepsilon)+\delta(r \varepsilon)+\delta(N \varepsilon)\}+\left|\int_{\lambda(y)}^{x} \omega_{2}(s, \varphi(s, y, \varepsilon), y) d s\right| \\
&=\mu(\varepsilon)+\varrho(\varepsilon)+\left|\int_{\lambda(y)}^{x} \omega_{2}(s, \varphi(s, y, \varepsilon), y) d s\right| \\
&=\nu(\varepsilon)+\left|\int_{\lambda(y)}^{x} \omega_{2}(s, \varphi(s, y, \varepsilon), y) d s\right|=\varphi(x, y, \varepsilon) .
\end{aligned}
$$

Ainsi nous arons établi que l'ensemble obtenu de l'ensemble $Z$ au moyen de la transformation (2.5) est un sous-ensemble de $Z$. La continuité de la transformation (2.5) résulte de la continuité uniforme des fonctions $F, G, H$ en vertu du fait que la convergence de la suite $\left\{C^{(k)}(x, y)\right\}$ dans l'espace $C^{\mathbf{1}}$ équivaut à la convergence uniforme des suites $\left\{U^{(k)}(x, y)\right\},\left\{U_{x}^{(k)}(x, y)\right\},\left\{U_{y}^{(k)}(x, y)\right\}$. On vérifie facilement que l'ensemble $Z$ est convexe (cf. (4.1)-(4.7)). Il est aussi compact. En effet, les fonctions $U(x, y)$ appartenant à l'ensemble $Z$ sont bornées en commun (cf. (4.1)) ainsi que leurs dérivées partielles (cf. (4.2), (4.3)) qui sont en outre équicontinues (cf. (4.4)-(4.7) et (4.12), (4.16)). Le théorème de Schauder peut donc être appliqué ([3], Satz 1, p. 173) et l'équation (2.1) possède au moins une solution de classe $C^{1}$ dans le rectangle $D$. En vertu du Lemme 1 (cf. §2) le Théorème 1 se trouve ainsi démontré.

§ 5. Remarque 3. L'Hypothèse $K$ est satisfaite lorsque la fonction $F(x, y, U, P, Q)$ satisfait à la condition de Lipschitz par rapport aux variables $P$ et $Q$. La propriété inverse n'a pas lieu.

En effet, on vérifie facilement que l'Hypothèse $K$ est satisfaite lorsque la fonction $F$ satisfait à la condition (3.6) arec $\omega_{1}(y, v, x)$ $\equiv \omega_{2}(x, v, y) \equiv \omega(v)$ où $\omega(v)$ est une fonction continue pour $v \geqslant 0$, non décroissante, positive pour $v>0$ et telle que $\omega(0)=0$ et que l'intégrale $\int_{0}^{e}(1 / \omega(v)) d v$ est divergente (cf. [2], p. 20). II est évident que cette dernière hypothèse est essentiellement plus générale que celle où la fonction $F(x, y, U, P, Q)$ satisfait à la condition de Lipschitz par rapport à $P$ et $Q$.

Admettant l'hypothèse de Lipschitz on peut remplacer dans le Théorème 1 l'hypothèse que la condition $a$ ou $b$ est satisfaite par des conditions moins spéciales. Cette propriété résulte du Théorème 2 qui suit.

Annales Polonis Mathematici IV. 
THÉоR亡̀me 2. Admettons l'Hypothèse $\mathrm{A}_{\mathrm{I}}$ (cf. §3) et supposons que les fonctions $F, G, H$ satisfassent à des conditions de Lipschitz

(5.1) $|F(x, y, U, P, Q)-F(x, y, U, \tilde{P}, \tilde{Q})| \leqslant M\{|P-\tilde{P}|+|Q-\tilde{Q}|\}$,

$$
|G(x, U, Q)-G(x, U, \tilde{Q})| \leqslant K|Q-\tilde{Q}|,
$$

$$
|H(y, U, P)-H(y, U, \tilde{P})| \leqslant K|P-\tilde{P}|
$$

dans leurs domaines d'existence et que
(5.4)
$h M+K<1$

oì h est defini par (3.4).

Dans ces hypothèses il existe une solution du Problème I.

Démonstration. La démonstration du Théorème 2 au fond analogue à celle du Théorème 1 , en est plus simple. Nous nous bornerons donc à l'esquisser. Considérons (cf. Remarque 2) dans l'espace $O^{\mathbf{1}}$ l'ensemble $Z^{*}$ des fonctions $U(x, y)$ de classe $C^{1}$ dans le rectangle $D$ (ef. (1.1)) satisfaisant aux relations (cf. (3.1) et (3.5))

$$
\begin{aligned}
& \text { (5.5) } \quad|U(x, y)| \leqslant r, \quad\left|U_{x}(x, y)\right| \leqslant r, \quad\left|U_{y}(x, y)\right| \leqslant r \\
& \text { (5.6) }\left|D_{x}(x, y+\varepsilon)-U_{x}(x, y)\right| \leqslant N \varepsilon \\
& \text { lorsque } \quad(x, y) \epsilon D, \quad(x, y+\varepsilon) \epsilon D, \quad \varepsilon \geqslant 0, \\
& \text { (5.7) }\left|U_{\boldsymbol{y}}(x+\varepsilon, y)-U_{y}(x, y)\right| \leqslant N_{\varepsilon} \\
& \text { lorsque } \quad(x, y) \in D, \quad(x+\varepsilon, y) \epsilon D, \quad \varepsilon \geqslant 0 \\
& \text { (5.8) }\left|U_{x}(x+\varepsilon, y)-U_{x}(x, y)\right| \leqslant \psi(\varepsilon) \\
& \text { lorsque } \quad(x, y) \in D, \quad(x+\varepsilon, y) \in D, \quad \varepsilon \geqslant 0 \\
& \text { (5.9) }\left|U_{y}(x, y+\varepsilon)-\bar{U}_{y}(x, y)\right| \leqslant \psi(\varepsilon) \\
& \text { lorsque } \quad(x, y)^{\prime} \in D, \quad(x, y+\varepsilon) \epsilon D, \quad \varepsilon \geqslant 0,
\end{aligned}
$$

où $\psi(\varepsilon)$ est une fonction convenablement choisie qui tend vers zéro avec $\varepsilon$. Avant de donner sa définition nous allons introduire quelques fonctions auxiliaires.

Soit $\delta(\varepsilon)$ le module de continuité (cf. le renvoi $\left({ }^{4}\right)$ ) commun aux fonctions $F, G, H, \gamma, \lambda$ dans leurs domaines d'existence. $\delta(\varepsilon)$ est donc une fonction non décroissante, non négative et tend vers zéro avec $\varepsilon$. Sans restreindre la généralité on peut admettre que $\delta(\varepsilon)$ est définie et bornée pour tous les $\varepsilon$ non négatifs. Il en résulte (cf. (5.4)) que la fonction $\eta(\varepsilon)$, définie par la formule

$$
\text { (5.10) } \begin{aligned}
\eta(\varepsilon)=\frac{1}{1-h M}\{K N \varepsilon+\delta(\varepsilon)+\delta(r \varepsilon+ & r \delta(\varepsilon))+N \delta(\varepsilon)+ \\
& +h[\delta(\varepsilon)+\delta(r \varepsilon)+N M \varepsilon]\}
\end{aligned}
$$

est non décroissante, non négative et bornée pour tout $\varepsilon \geqslant 0$ et qu'elle tènd vers zéro avec $\varepsilon$,
Posons

$$
\begin{gathered}
\psi^{(1)}(\varepsilon)=\eta(\varepsilon), \\
\psi^{(s+1)}(\varepsilon)=\eta(\varepsilon)+\frac{K}{1-h \mathbb{M}} \psi^{(s)}(\delta(\varepsilon)), \quad s=1,2, \ldots
\end{gathered}
$$

En vertu de (5.4) la suite $\left\{\psi^{(s)}(\varepsilon)\right\}$ est uniformément convergente. On vérifie facilement que la limite de cette suite

$$
\psi(\varepsilon)=\lim _{s \rightarrow \infty} \psi^{(s)}(\varepsilon)
$$

est une fonction non décroissante, non négative et satisfait aux relations

$$
\begin{gathered}
\psi(\varepsilon) \rightarrow 0 \quad \text { lorsque } \quad \varepsilon \rightarrow 0, \\
\psi(\varepsilon)=\eta(\varepsilon)+\frac{K}{1-\hbar M} \psi(\delta(\varepsilon)) .
\end{gathered}
$$

La fonction $\psi(\varepsilon)$ étant ainsi définie ( $\left.{ }^{\circ}\right)$, nous allons démontrer que (2.5) transforme l'ensemble $Z^{*}$ (cf. (5.5)-(5.9)) en un sous-ensemble de celui-ci. En vertu de l'Hypothèse $A_{\mathrm{I}}$ la fonction $\tilde{U}(x, y)$ définie par (2.5) satisfait aux inégalités (5.5)-(5.7) (cf. (4.19), (4.20)). En tenant compte de (5.5), (5.2), (5.7), (5.9), on démontre que

(5.14) $\mid G\left[x+\varepsilon, \delta(x+\varepsilon, \gamma(x+\varepsilon)), J_{y}(x+\varepsilon, \gamma(x+\varepsilon))\right]-$

$$
\begin{aligned}
-G\left[x, U(x, \gamma(x)), U_{y}(x, \gamma(x))\right] \mid \\
\leqslant \delta(\varepsilon)+\delta(r \varepsilon+r \delta(\varepsilon))+K N \varepsilon+K \psi(|\gamma(x+\varepsilon)-\gamma(x)|) \\
\leqslant \delta(\varepsilon)+\delta(r \varepsilon+r \delta(\varepsilon))+K N \varepsilon+K \psi(\delta(\varepsilon))
\end{aligned}
$$

et en s'appuyant encore sur (5.1) et (5.8) on établit l'inégalité

(5.15) $\quad \mid F\left[x+\varepsilon, t, U(x+\varepsilon, t), \delta_{x}(x+\varepsilon, t), \sigma_{y}(x+\varepsilon, t)\right]-$

$$
-F\left[x, t, U(x, t), D_{x}(x, t), U_{y}(x, t)\right] \mid
$$

$$
\leqslant \delta(\varepsilon)+\delta(r \varepsilon)+M \psi(\varepsilon)+M N \varepsilon .
$$

Il résulte de (4.19), en vertu de (5.14), (3.5), (5.15), (3.4), (5.10) et (5.13), que

$$
\begin{aligned}
\left|\tilde{U}_{x}(x+\varepsilon, y)-\tilde{U}_{x}(x, y)\right| \leqslant & \delta(\varepsilon)+\delta(r \varepsilon+r \delta(\varepsilon))+K N_{\varepsilon}+K \psi(\delta(\varepsilon))+ \\
& +N \delta(\varepsilon)+h\{\delta(\varepsilon)+\delta(r \varepsilon)+M \psi(\varepsilon)+M N \varepsilon\} \\
= & (1-h M) \eta(\varepsilon)+K \psi(\delta(\varepsilon))+h M \psi(\varepsilon)=\psi(\varepsilon) .
\end{aligned}
$$

(ब) Dans l'hypothèse supplémentaire que

$|\gamma(\dot{x})-\gamma(\bar{x})| \leqslant m|x-\bar{x}|, \quad|\lambda(y)-\lambda(\bar{y})| \leqslant m|y-\bar{y}|, \quad h M+m K<1, \quad m \geqslant 1$, on peut poser tout simplement $\psi(\varepsilon)=(1-\hbar M) \eta(\varepsilon) /(1-\hbar M-K m)$ où $\eta(\varepsilon)$ est donné par (5.10) (en admettant que $\delta(b \varepsilon) \leqslant b \delta(\varepsilon)$ pour chaque $b \geqslant 1$ ce qu'on peut toujours faire sans restreindre la généralité). 
Une démonstration tout à fait analogue de l'inégalité

$$
\left|\tilde{U}_{y}(x, y+\varepsilon)-\tilde{U}_{y}(x, y)\right| \leqslant \psi(\varepsilon)
$$

peut être omise ici.

Ainsi nous avons établi que l'ensemble obtenu de l'ensemble $Z^{*}$ au moyen de la transformation (2.5) est un sous-ensemble de $Z^{*}$. On vérifie facilement (cf. la démonstration du Théorème 1), que la transformation (2.5) est continue dans $Z^{*}$ et que l'ensemble $Z^{*}$ est convexe et compact. Le théorème de Schauder pouvant être appliqué, l'équation (2.1) (cf. Remarque 2) possède au moins une solution de classe $C^{1}$ dans le rectangle $D$. En vertu du Lemme 1 le Théorème 2 se trouve ainsi démontré.

Remarque 4. Les hypothèses du Théorème 2 concernant les fonctions $G(x, U, Q)$ et $H(y, U, P)$ (ef. (5.2) - (5.4) et Hypothèse $A_{I}, 4^{0}$ ) ne sont pas les plus faibles possibles. Nous les arons admises pour simplifier la démonstration de notre théorème. Toutefois elles ne peurent pas être supprimées. En effet, dans le cas où $n=1, F \equiv 0, \gamma(x) \equiv x$, $\lambda(y) \equiv-y, \alpha=\beta, G \equiv-Q, H \equiv P+y$, le Problème I n'admet pas de solution (même locale) quels que soient le vecteur constant $\dot{U}$ et le point $\left(x_{0}, y_{0}\right) \in D$ (cf. (1.1)). Dans cet example, étroitement lié à l'exemple donné dans la Remarque 4 de la note [4], l'inégalité (5.4) et Hypothèse A, $4^{\circ}$ ne sont pas satisfaites.

§ 6. Existence d'une solution du Problème II. Dans le cas, où $\lambda(y)$ est constante, ou $B(y, U, P)-\lambda^{\prime}(y) P$ ne dépend pas de $P, l^{\prime}$ existence d'une solution du Problème II résulte du Théorème 1 , cas $b$ (cf. Remarque 1). En nous dispensant d'énoncer ce corollaire, passons à un autre théorème assurant l'existence d'une solution du Problème II. On vérifie facilement que l'existence d'une solution du Problème II résulte de celle d'une solution de classe $C^{1}$ du système

$$
U=\mathscr{L}[U]
$$

où l'opérateur $\mathscr{E}[U]$ est donné par la formule

$$
\begin{aligned}
\mathcal{L}[U]=\dot{U} & +\int_{\nu_{0}}^{y} B\left[t, U(\lambda(t), t), U_{x}(\lambda(t), t)\right] d t+ \\
& +\int_{\lambda(\nu)}^{x} G\left[s, U(s, \gamma(s)), U_{y}(s, \gamma(s))\right] d s+ \\
& +\int_{\lambda(\nu)}^{x}\left\{\int_{\gamma(s)}^{y} F\left[s, t, U(s, t), U_{x}(s, t), U_{\nu}(s, t)\right] d t\right\} d s .
\end{aligned}
$$

En tenant compte des notations introduites au commencement du $\S 3$ nous énoncerons l'hypothèse suivante:

HYPOTHìse $A_{\text {II }} 1^{0}-\beta \leqslant y_{0} \leqslant \beta$.

$2^{\circ} \gamma(x)$ est une fonction continue telle que

$$
-\beta \leqslant \gamma(x) \leqslant \beta \quad \text { lorsque } \quad-\alpha \leqslant x \leqslant \alpha,
$$

$\lambda(y)$ est une fonction de classe $C^{1}$ telle que

$$
-\alpha \leqslant \lambda(y) \leqslant \alpha \quad \text { lorsque } \quad-\beta \leqslant y \leqslant \beta ; \quad
$$

$w$ est un nombre non négatif tel que

$$
-w \leqslant \lambda^{\prime}(y) \leqslant w .
$$

$3^{\circ}$ La fonction $F(x, y, U, P, Q)$, continue dans l'ensemble $\Pi$ defini par (3.1), y satisfait à la condition

où

$$
N \leqslant \min \left\{r / h^{2}, r / h(w+1)\right\}
$$

$$
N_{0}=\max _{I}|F| \text {. }
$$

$4^{\circ}$ Le vecteur constant $\dot{U}$ et les fonctions vectorielles continues $G, B$ satisfont aux inégalités: $|\stackrel{\cup}{U}| \leqslant 0^{*},|G(x, \sigma, Q)| \leqslant c^{*}$ dans l'ensemble (3.2), $|B(y, U, P)| \leqslant e^{*}$ dans l'ensemble (3.3), où (cf. (6.3) et (6.4))

$$
e^{*}=\min \left\{\left(r-N h^{2}\right) /(1+2 h),(r-w N h-N h) /(1+w)\right\} .
$$

THÉonìme 3. Admettons les hypothèses $\mathbf{K}$ et $\mathbf{A}_{\text {II }}$ et supposons vérifiée l'hypothèse suivante:

La fonction $G$ ne dépend pas de la variable $Q$ ou bien la fonction $\gamma(x)$ est constante.

Dans ces hypothèses il existe une solution du Problème $\Pi\left({ }^{7}\right)$.

Démonstration (esquisse). Soit $\mathfrak{Z}$ l'ensemble des fonctions $U(x, y)$ de classe $C^{1}$ dans le rectangle $D$ (cf. (1.1)) qui satisfont aux inégalités (4.1)-(4.7), où $\psi(y, x, \varepsilon)$ est une fonction définie dans le $\S 4$ et $\varphi(x, y, \varepsilon)$ est une fonction convenablement choisie. Avant de donner sa définition, différente de celle de la fonction $\varphi$ considerée dans le $\S 4$, nous allọns introduire quelques fonctions auxiliaires. Soit $\delta(\varepsilon)$ le module de conti-

(7) Cette solution satisfait évidemment aux relations:

$$
|\delta(x, y)| \leqslant r, \quad\left|U_{x}(x, y)\right| \leqslant r, \quad\left|J_{y}(x, y)\right| \leqslant r, \quad\left|U_{x y}(x, y)\right| \leqslant N
$$

dans le reotangle $D$ (of. (1.2) et Hypothèse $A_{\text {II }} 3^{\circ}$ ). 
nuité commun aux fonctions $F(x, y, U, P, Q), G(x, U, Q), B(y, U, P)$, $\gamma(x), \lambda^{\prime}(y)$ dans leurs domaines d'existence (cf. le renvoi $\left.\left(^{4}\right)\right)$. Posons

$$
\tau(\varepsilon)=\sup _{\substack{-\beta \leqslant t \leqslant \beta \\-\beta \leqslant y \leqslant \beta}} \omega_{1}[t, \psi(t, \lambda(y),|\lambda(y+\varepsilon)-\lambda(y)|), \lambda(y)],
$$

(6.6) $\quad \sigma^{*}(\varepsilon)=\max \{\delta(w \varepsilon)+\delta(r w \varepsilon+r \delta(w \varepsilon)), \delta(w \varepsilon)+\delta(r w \varepsilon)+\delta(N w \varepsilon)\}$

et soit

$$
\begin{aligned}
(6.7) \nu^{*}(\varepsilon)= & \delta(\varepsilon)+\delta(r \varepsilon+r w \varepsilon)+\varrho(\varepsilon)+c^{*} \delta(\varepsilon)+w \sigma^{*}(\varepsilon)+h N \delta(\varepsilon)+ \\
& +w\{N \varepsilon+N \delta(w \varepsilon)+h[\delta(w \varepsilon)+\delta(r w \varepsilon)+\delta(N w \varepsilon)+\tau(\varepsilon)]\}+ \\
& +w N \varepsilon+h[\delta(\varepsilon)+\delta(r \varepsilon)+\delta(N \varepsilon)],
\end{aligned}
$$

où $\varrho(\varepsilon)$ est défini par (4.13).

Désignant par $\varkappa_{1}(x, \xi, \eta ; y)$ et $x_{2}(x, \xi, \eta ; y)$ les mêmes fonctions que dans le $\S 4$, posons formellement

$$
\varphi(x, y, \varepsilon)=\left\{\begin{array}{lll}
x_{1}\left(x, \lambda(y), v^{*}(\varepsilon) ; y\right) & \text { lorsque } \quad x \geqslant \lambda(y), \\
x_{2}\left(x, \lambda(y), \nu^{*}(\varepsilon) ; y\right) & \text { lorsque } x<\lambda(y) .
\end{array}\right.
$$

On vérifie facilement que la fonction $\varphi(x, y, \varepsilon)$ est définie pour $(x, y) \epsilon D$ et $\varepsilon$ suffisamment petit et que les relations suivantes subsistent

$$
\varphi(x, y, \varepsilon) \Rightarrow 0 \quad \text { dans le rectangle } D
$$

$$
\left|\int_{\lambda(\nu)}^{x} \omega_{2}(s, \varphi(s, y, \varepsilon), y) d s\right|=\varphi(x, y, \varepsilon)-\nu^{*}(\varepsilon) .
$$

Nous allons démontrer que la transformation

(6.10)

$$
\tilde{U}(x, y)=\mathcal{L}[U(x, y)]
$$

où $\mathcal{L}[U]$ est défini par (6.2), transforme l'ensemble $\mathcal{E}$ en un sous-ensemble de celui-ci.

En effet il résulte des relations (cf. le renvoi (s))

$\tilde{U}_{x}(x, y)=G\left[x, U(x, \gamma(x)), U_{y}(x, \gamma(x))\right]+$

(6.11)

$$
+\int_{\gamma(x)}^{y} F\left[x, t, U(x, t), U_{x}(x, t), U_{v}(x, t)\right] d t
$$

$\tilde{U}_{\boldsymbol{\nu}}(x, y)=B\left[y, U(\lambda(y), y), U_{x}(\lambda(y), y)\right]-$

$-\lambda^{\prime}(y) G\left[\lambda(y), U(\lambda(y), \gamma(\lambda(y))), U_{y}(\lambda(y), \gamma(\lambda(y)))\right]-$

$-\lambda^{\prime}(y) \int_{\gamma(\lambda(y))}^{y} F\left[\lambda(y), t, U(\lambda(y), t), U_{x}(\lambda(y), t), D_{y}(\lambda(y), t)\right] d t+$

$+\int_{\lambda(y)}^{x} F\left[s, y, U(s, y), U_{x}(s, y), U_{y}(s, y)\right] d s$ que $\tilde{U}(x, y)$ est une fonction de classe $O^{\mathbf{1}}$ dans le rectangle $D$ et qu'elle satisfait aux relations (4.1)-(4.5) (cf. Hypothèse $A_{\text {II }}$ ). La démonstration que la fonction $\tilde{U}(x, y)$ satisfait aussi à l'inégalité (4.6) est la même que dans le cas du Théorème 1. Pour démontrer qu'elle satisfait à l'inégalité (4.7), on profite des relations suivantes assez faciles à vérifier:

$$
|\lambda(y+\varepsilon)-\lambda(y)| \leqslant w \varepsilon
$$

(cf. $(6.3))$,

(6.12) $\quad \mid B\left[y+\varepsilon, U(\lambda(y+\varepsilon), y+\varepsilon), U_{x}(\lambda(y+\varepsilon), y+\varepsilon)\right]-$

$$
-B\left[y, U(\lambda(y), y), U_{x}(\lambda(y), y)\right] \mid
$$

$$
\leqslant \delta(\varepsilon)+\delta(r \varepsilon+r w \varepsilon)+\delta(N \varepsilon+\psi(y, \lambda(y),|\lambda(y+\varepsilon)-\lambda(y)|))
$$

$$
\leqslant \delta(\varepsilon)+\delta(r \varepsilon+r w \varepsilon)+\varrho(\varepsilon),
$$

où $\varrho(\varepsilon)$ est défini par (4.13),

(6.13) $\quad \mid G\left[\lambda(y+\varepsilon), U(\lambda(y+\varepsilon), \gamma(\lambda(y+\varepsilon))), J_{y}(\lambda(y+\varepsilon), \gamma(\lambda(y+\varepsilon)))\right]-$

(cf. (6.6)),

$$
-G\left[\lambda(y), U(\lambda(y), \gamma(\lambda(y))), \sigma_{y}(\lambda(y), \gamma(\lambda(y)))\right] \mid \leqslant \sigma^{*}(\varepsilon),
$$

(6.14) . $F\left[\lambda(y+\varepsilon), t, U(\lambda(y+\varepsilon), t), \delta_{x}(\lambda(y+\varepsilon), t), \sigma_{y}(\lambda(y+\varepsilon), t)\right]-$ $-F\left[\lambda(y), t, \sigma(\lambda(y), t), \sigma_{x}(\lambda(y), t), \sigma_{y}(\lambda(y), t)\right] \mid$ $\leqslant \delta(w \varepsilon)+\delta(r w \varepsilon)+\delta(N w \varepsilon)+\omega_{1}[t, \psi(t, \lambda(y),|\lambda(y+\varepsilon)-\lambda(y)|), \lambda(y)]$ $\leqslant \delta(w \varepsilon)+\delta(r w \varepsilon)+\delta(N w \varepsilon)+\tau(\varepsilon)$

(cf. (6.5)),

(6.15) $\mid F\left[s, y+\varepsilon, U(s, y+\varepsilon), U_{x}(s, y+\varepsilon), U_{y}(s, y+\varepsilon)\right]-$

$-F\left[s, y, U(s, y), U_{x}(s, y), U_{y}(s, y)\right] \mid$

$\leqslant \delta(\varepsilon)+\delta(r \varepsilon)+\delta(N \varepsilon)+\omega_{2}(s, \varphi(s, y, \varepsilon), y)$.

En vertu des relations (6.11)-(6.15), (6.7) et (6.9) on démontre facilement (cf. Hypothèse $A_{\text {III }}$ ) que

$$
\begin{aligned}
& \left|\tilde{U}_{y}(x, y+\varepsilon)-\tilde{U}_{y}(x, y)\right| \leqslant \delta(\varepsilon)+\delta(r \varepsilon+r w \varepsilon)+\varrho(\varepsilon)+c^{*} \delta(\varepsilon)+ \\
& +w \sigma^{*}(\varepsilon)+N h \delta(\varepsilon)+w\{N \varepsilon+N \delta(w \varepsilon)+h[\delta(w \varepsilon)+\delta(r w \varepsilon)+\delta(N w \varepsilon)+\tau(\varepsilon)]\}+ \\
& +w N \varepsilon+h\{\delta(\varepsilon)+\delta(r \varepsilon)+\delta(N \varepsilon)\}+\left|\int_{\lambda(\nu)}^{x} \omega_{2}(s, \varphi(s, y, \varepsilon), y) d s\right| \\
& \therefore \leqslant v^{*}(\varepsilon)+\left|\int_{\lambda(\nu)}^{x} \omega_{2}(s, \varphi(s, y, \varepsilon), y) d s\right|=\varphi(x, y, \varepsilon) .
\end{aligned}
$$


Ainsi nous avons établi que l'ensemble obtenu de l'ensemble $\mathfrak{Z}$ au moyen de la transformation (6.10) est un sous-ensemble de $\mathfrak{Z}$. On vérifie facilement que l'ensemble $\mathfrak{Z}$ est convexe et compact (cf. (4.12) et (6.8)) et que la transformation (6.10) est continue. Le théorème de Schauder (cf. [3], Satz 1, p. 173) pouvant être appliqué, l'équation (6.1) possède au moins une solution de classe $C^{1}$ dans le rectangle $D$. Le Théorème 3 se trouve ainsi démontré.

Nous énoncerons encore un autre théorème sur l'existence d'une solution du Problème II, analogue au Théorème 2 relatif au Problème $\mathrm{I}$.

THÉoRł̀me 4. Admettons l'Hypothèse $\mathrm{A}_{\mathrm{II}}$, les relations (5.1), (5.2) et supposons que $|B(y, U, P)-B(y, U, \tilde{P})| \leqslant K^{*}|P-\tilde{P}|$ dans l'ensemble (3.3) et que les constantes de Lipschitz $M, K$ et $K^{*}$ soient suffisamment petites.

Dans ces hypothèses le Problème II possède une solution.

§ 7. Désignons par $\Pi_{1}$ l'ensemble défini (dans l'espace de variables $x, y, U, P, Q)$ par les relations

(7.1) $-\alpha \leqslant x \leqslant \alpha, \quad-\beta \leqslant y \leqslant \beta, \quad$ où $\quad 0<\alpha<\infty, \quad 0<\beta<\infty$

( $U, P, Q$ arbitraires) et considérons en outre les ensembles $\Sigma_{1}$ et $\Sigma_{2}$ définis par les relations

$$
\begin{array}{ll}
-\alpha \leqslant x \leqslant \alpha, & \quad, Q \text { arbitraires, } \\
-\beta \leqslant y \leqslant \beta, & \quad, P \text { arbitraires. }
\end{array}
$$

HYPOTHÈSE $\mathrm{R}_{\mathrm{I}}\left(\mathrm{R}_{\mathrm{II}}\right)$. La fonction $F(x, y, U, P, Q)$ est continue ef bornée dans l'ensemble (7.1) et les fonctions $G(x, U, Q), H(y, U, P)$ $(G(x, U, Q), B(y, U, P))$ le sont dans les ensembles (7.2) et (7.3) respectivement.

Remarque 5. On peut substituer dans les théorèmes 1 et 2 (3 et 4 ) les ensembles (7.1), (7.2), (7.3) au lieu des ensembles (3.1), (3.2) et (3.3) en remplaçant en même temps les prémisses $3^{\circ}$ et $4^{\circ}$ de $1^{\prime}$ Hypothèse $A_{I}$ (AII) par l'Hypothèse $\mathrm{R}_{\mathrm{I}}\left(\mathrm{R}_{\mathrm{II}}\right)$.

La solution $U(x, y)$ du Problème I satisfait alors aux relations (cf. les renvois $\left({ }^{3}\right)$ et $\left.\left({ }^{7}\right)\right)$

$$
\begin{gathered}
|U(x, y)| \leqslant r_{1}, \quad\left|U_{x}(x, y)\right| \leqslant r_{1}, \quad\left|U_{y}(x, y)\right| \leqslant r_{1}, \\
\left.\left|U_{x y}(x, y)\right| \leqslant N \quad \text { dans } D, \text { où (cf. }(3.4)\right) \\
r_{1}=\max \left\{c_{1}+h N, c_{1}(1+2 h)+N h^{2}\right\}, \\
c_{1}=\max \left\{\sup _{\Sigma_{1}}|G|, \sup _{\Sigma_{2}}|H|,|\stackrel{\circ}{U}|\right\}, \quad N=\sup _{\pi_{1}}|F|,
\end{gathered}
$$

tandis que la solution $U(x, y)$ du Problème II satisfait aux relations

$$
|U(x, y)| \leqslant r_{2}, \quad\left|U_{x}(x, y)\right| \leqslant r_{2}, \quad\left|U_{y}(x, y)\right| \leqslant r_{2}, \quad\left|U_{x y}(x, y)\right| \leqslant N
$$

dans le rectangle $D$ où (cf. (3.4) et (6.3))

$$
\begin{aligned}
& r_{2}=\max \left\{N h^{2}+(1+2 h) c_{2},(w+1)\left(N h+c_{2}\right)\right\}, \\
& c_{2}=\max \left\{\sup _{\Sigma_{\mathbf{1}}}|G|, \sup _{\Sigma_{\mathbf{2}}}|B|,|\dot{U}|\right\}, \quad N=\sup _{\boldsymbol{M}_{\mathbf{1}}}|F| .
\end{aligned}
$$

La Remarque 5 résulte du fait que en vertu de l'Hypothèse $R_{I}\left(R_{I I}\right)$ les prémisses $3^{\circ}$ et $4^{\circ}$ de l'Hypothèse $A_{I}\left(A_{\text {II }}\right)$ sont satisfaites lorsque $r=r_{1}, c=c_{1}\left(r=r_{2}, e=c_{2}\right)$.

§ 8. Existence de solutions des problèmes classiques. Voici maintenant les Corollaires 1 et 2 qui résultent immédiatement du Théorème 1 ainsi que du Théorème 3 .

CoRollaIRE 1. Existence d'une solution du problème de Darboux. Admettons l'Hypothèse $\mathrm{K}$ (cf. § 3 ) relativement à la fonction $F(x, y, U, P, Q)$ continue dans l'ensemble II défini par les inégalités (3.1). Supposons que les fonctions $\sigma(x)$ et $\tau(y)$ soient des fonctions de classe $C^{1}$ dans les intervalles $\langle-\alpha, \alpha\rangle$ et $\langle-\beta, \beta\rangle$ respectivement et que $\sigma(0)=\tau(0)$. Dans l'hypothèse que les fonctions $|F(x, y, U, P, Q)|,\left|\sigma^{\prime}(x)\right|,|\tau(y)|,\left|\tau^{\prime}(y)\right|$ sont bornées par des constantes suffisamment petites il existe une solution $U(x, y) d u$ système (1.3) ayant des dérivées $U_{x}, U_{y}, U_{x y}$ continues dans le rectangle $D$ (cf. (1.1)) et satisfaisant aux relations

$$
\begin{array}{lll}
U(x, 0)=\sigma(x) & \text { lorsque } & -\alpha \leqslant x \leqslant \alpha, \\
U(0, y)=\tau(y) & \text { lorsque } & -\beta \leqslant y \leqslant \beta .
\end{array}
$$

On a évidemment (cf. (3.5))

(8.1) $|U(x, y)| \leqslant r, \quad\left|U_{x}(x, y)\right| \leqslant r, \quad\left|U_{y}(x, y)\right| \leqslant r, \quad\left|U_{x y}(x, y)\right| \leqslant N$ dans le rectangle $D$. Dans le cas où la fonction $F$ est bornée dàns l'ensemble $\Pi_{1}$ défini par les relations (7.1) les fonctions $\sigma(x)$ et $\tau(y)$ de classe $C^{1}$ dans les intervalles $\langle-\alpha, \alpha\rangle$ et $\langle-\beta, \beta\rangle$ respectivement ne sont soumises qu'à la condition $\sigma(0)=\tau(0)$. Les relations $(8,1)$ subsistent pour un $r$ convenablement choisi $\left({ }^{8}\right)$.

Corollarre 2. Existence d'une solution du problème de Cauchy. Admettons l'Hypothèse $\mathrm{K}$ (cf. § 3) relativement à la fonotion $I^{\prime}(x, y, U, P, Q)$ continue dans l'ensemble $\Pi$ défini par les inégalités (3.1).

(8) Il suffit d'admettre $r=r_{1}$, où $r_{1}$ est défini par (7.4), $o_{1}=\max \{|\tau(0)|$, $\left.\max \left|\sigma^{\prime}(x)\right|, \quad \max \left|\tau^{\prime}(y)\right|\right\}, \quad N=\sup \left|F^{\prime}\right|, h=2 \max (\alpha, \beta)$. 
Supposons que $\varrho(x)$ soit une fonction continue, $\sigma(x), \gamma(x)$ soient des fonctions de classe $C^{1}$ dans l'intervalle $\langle-a, a\rangle$ et

$$
\min _{-a<x \leqslant a} \gamma(x)=-\beta, \quad \max _{-a<x<a} \gamma(x)=\beta, \quad \gamma^{\prime}(x) \neq 0 .
$$

Alors, dans l'hypothèse que les fonctions $|F(x, y, U, P, Q)|,|\sigma(x)|,\left|\sigma^{\prime}(x)\right|$, $|\varrho(x)|$ sont bornées par des constantes suffisamment petites $\left(^{{ }^{\circ}}\right.$ il existe une solution du système (1.3) ayant des dérivées $U_{x}, U_{y}, U_{x y}$ continues dans le rectangle $D$ "et satisfaisant aux relations

$$
\begin{array}{cll}
U_{x}(x, \gamma(x))=\varrho(x) & \text { lorsque } & -\alpha \leqslant x \leqslant \alpha, \\
U(x, \gamma(x))=\sigma(x) & \text { lorsque } & -\alpha \leqslant x \leqslant \alpha .
\end{array}
$$

Passons maintenant au. Corollaire 3 qui résulte immédiatement $\mathrm{du}$ Théorème 3.

Corollarre 3. Existence d'une solution du problème de Picard. Admettons l'hypothèse $\mathrm{K}$ relativement à la fonction $\mathbb{F}(x, y, \boldsymbol{U}$, $P, Q)$ continue dans l'ensemble $\Pi$ défini par les inégalités (3.1). Supposons que les fonctions $\sigma(x)$ et $\tau(y), \lambda(y)$ soient des fonctions de classe $C^{\mathbf{1}}$ dans les intervalles $-\alpha \leqslant x \leqslant \alpha$ et $-\beta \leqslant y \leqslant \beta$ respectivement et que

$$
\lambda(-\beta)=-\alpha, \quad \sigma(-\alpha)=\tau(-\beta), \quad-\alpha \leqslant \lambda(y) \leqslant \alpha
$$

$$
\text { lorsque }-\beta \leqslant y \leqslant \beta \text {. }
$$

Alors si l'on admet que les fonctions $|F(x, y, U, P, Q)|,|\tau(y)|,\left|\tau^{\prime}(y)\right|$, $\left|\sigma^{\prime}(x)\right|$ sont bornées par des constantes suffisamment petites (cf. lo renvoi $\left({ }^{9}\right)$ ), il existe une solution du système (1.3) ayant des dérivées $U_{x}, U_{y}, U_{x y}$ continues dans le rectangle $D$ et satisfaisant aux relations

$$
\begin{array}{lll}
U(x,-\beta)=\sigma(x) & \text { lorsque } & -\alpha \leqslant x \leqslant \alpha, \\
U(\lambda(y), y)=\tau(y) & \text { lorsque } & -\beta \leqslant y \leqslant \beta .
\end{array}
$$

Voici encore le Corollaire 4, déduit par A. Lasota du Théorème 2.

Corollatre 4. Existence locale d'une solution du probleme de Goursat. Admettons (5.1) et les prémisses $2^{\circ}$ et $3^{\circ}$ de l'hypothèse $\mathbf{A}_{\mathrm{I}}$. Supposons que les fonctions $\sigma(x), \gamma(x), \tau(y), \lambda(y)$ soient des fonctions de classe $C^{1}$ et que $\left|\gamma^{\prime}(x)\right|<1$ lorsque $-\alpha \leqslant x \leqslant \alpha,\left|\lambda^{\prime}(y)\right|<1$ lorsque $-\beta \leqslant y \leqslant \beta, \gamma(0)=\lambda(0)=0, \sigma(0)=\tau(0)$. Alors si l'on admet que les constantes $\alpha, \beta,|\tau(0)|$ et les bornes supérieures des fonctions $\left|\tau^{\prime}(y)\right|,\left|\sigma^{\prime}(x)\right|$

(9) Cette hypothèse est superflue dans le cas où la fonction $F(x, y, U, P, Q)$ est continue et bornée dans l'ensemble $\Pi_{1}$ défini par les relations (7.1). La Remarque permet d'évaluer la solution $U(x, y)$ ainsi que ses dérivées $U_{x}, U_{y}, U_{x y}$ dans le rectan gle $D$. Nous nous dispensons de donner cette évaluation, analogue à celle qui a été faite dans le Corollaire 1 (cf. le renvoi $\left(^{(\theta)}\right)$. sont suffisamment petites, il existe une solution du système (1.3) ayant les dérivés $U_{x}, U_{y}, U_{x y}$ continues dans le rectangle $D$ et satisfaisant aux relations

$$
\begin{array}{lll}
U(x, \gamma(x))=\sigma(x) & \text { lorsque } & -\alpha \leqslant x \leqslant \alpha, \\
U(\lambda(y), y)=\tau(y) & \text { lorsque } & -\beta \leqslant y \leqslant \beta .
\end{array}
$$

8. Remarque 6. La démonstration des théorèmes 1 et 2 était basée sur la propriété que chaque point fixe de la transformation (2.5), considérée dans un ensemble de l'espace $\sigma^{1}$, est une-solution du Problème I (cf. \$2). Oñ peut aussi démontrer les théorèmes 1 et 2 en se servant d'une transformation (différente de (2.5)), définie dans un ensemble convenablement choisi dans l'espace $C$ et dont chaque point fixe est identique à la dérivée seconde mixte d'une solution du Problème I. C'est cette dernière méthode qui a été adaptée par $\mathrm{S}$. Mazur à la démonstration du résultat de $\mathrm{P}$. Hartman et $\mathrm{A}$. Wintner concernant l'existence d'une solution du problème de Darboux (cf. l'introduction). A savoir S. Mazur a démontré l'existence d'une solution continue $W(x, y)$ du système

$$
W(x, y)=F(x, y, U(x, y), P(x, y), Q(x, y)),
$$

où (cf. les notations introduites dans le Corollaire $1, \S 8$ )

$$
\begin{gathered}
U(x, y)=\sigma(x)+\tau(y)-\sigma(0)+\int_{0}^{x}\left\{\int_{0}^{y} W(s, t) d t\right\} d s \\
P(x, y)=\sigma^{\prime}(x)+\int_{0}^{y} W(x, t) d t, \quad Q(x, y)=\tau^{\prime}(y)+\int_{0}^{x} W(s, y) d s
\end{gathered}
$$

en appliquant le théorème de Schauder à la transformation

$$
T[W]=F(x, y, U(x, y), P(x, y), Q(x, y))
$$

et à un ensemble convenablement choisi dans l'espace $C$.

La méthode de S. Mazur peut aussi être appliquée à la démonstration des théorèmes 3 et 4 .

Remarque 7. La solution du Problème I, dont l'existence est assurée par les théorèmes énoncés dans cette note ne doit pas forcément être unique. Il en est ainsi même dans le cas où la fonction continue $F(x, y, U, P, Q)$ ne dépend pas des variables $x, y, P, Q$ et les fonctions $\gamma(x), \lambda(y), G(x, U, Q), H(y, U, P)$ sont constantes.

Cette remarque résulte d'un exemple dû à $\mathrm{P}$. Hartman et $\mathrm{A}$. Wintner ([1] (i), p. 841). Je me propose d'étudier dans une note à part l'existence d'une solution unique des Problèmes I et II. 


\section{Travaux cités}

[1] P. Hartman and A. Wintner, On hyperbolic partial differential equations, American Journal of Mathematics 74 (4) (1952), p. 834-864.

[2] E. Kamke, Differentialgleiohungen reeller Funletionen, Leipzig 1930.

[3] J. Schauder, Der Fixpunktsatz in Funktionalraümien, Studia Mathema. tica 2 (1930), p. 171-180.

[4] Z. Szmydt, Sur un nouvean type de problèmes pour un système d'équations différentielles hyperboliques du second ordre d̀ deux variables indépondantes, Bulletin de l'Académie Polonaise des Sciences, Cl. III. Vol. 4 (2) (1956), p. 67-72.

\section{Propriétés des intégrales de l'équation parabolique normale}

par W. Pogokzelskr (Warszawa)

1. Introduction. Soit l'équation aux dérivées partielles du type parabolique

(1) $\hat{\Psi}(u)=\sum_{a, \beta=1}^{n} a_{\alpha \beta}(A, t) \frac{\partial^{2} u}{\partial x_{\alpha} \partial x_{\beta}}+\sum_{a=1}^{n} b_{\alpha}(A, t) \frac{\partial u}{\partial x_{a}}+c(A, t) u-\frac{\partial u}{\partial t}=0$

où les coefficients $a_{\alpha \beta}(A, t), b_{a}(A, t), c(A, t)$ sont des fonctions déterminées dans la région fermée

$$
A\left(x_{1}, \ldots, x_{n}\right) \in \Omega+S, \quad 0 \leqslant t \leqslant T,
$$

$\Omega$ étant un domaine borné dans l'espace euclidien à $n(n \geqslant 2)$ dimensions, limité par la surface fermée $S$. On suppose que la forme quadratique

$$
\sum_{a, \beta=1}^{n} a_{a \beta}(A, t) X_{\alpha} X_{\beta}
$$

est définie positive dans la région (2).

La première idée de la recherche de la solution fondamentale de l'équation (1) est due à G. Giraud [1]. Ensuite F. Dressel [2] a étudié d'une façon complète la solution fondamentale de l'équation (1), mais sous l'hypothèse restrictive que les coefficients $a_{a \beta}$ soient des fonctions admettant des dérivées secondes. Récemment W. Pogorzelski [3], l'auteur de cet article, a étudié la solution fondamentale de l'équation (1) sous l'hypothèse plus générale suivante:

1. Les coefficients $a_{\alpha \beta}(A, t)$ vérifient dans la région (2) la condition de Hölder

$$
\left|a_{\alpha \beta}(A, t)-a_{\alpha \beta}\left(\dot{A_{1}}, t_{1}\right)\right|<k_{k}\left(r_{A A_{1}}^{h}+\left|t-t_{1}\right|^{h^{\prime}}\right)
$$

k etant une constante positive et $h, h^{\prime}$ deux constantes positives qui ne sont pas supérieures à l'unité, $r_{\mathcal{A}}$ désignant la distance des points $A, A_{1}$. 